\title{
STRONGLY LIFTABLE SCHEMES AND THE KAWAMATA-VIEHWEG VANISHING IN POSITIVE CHARACTERISTIC
}

\author{
QIHONG XIE
}

\begin{abstract}
A smooth scheme $X$ over a field $k$ of positive characteristic is said to be strongly liftable, if $X$ and all prime divisors on $X$ can be lifted simultaneously over $W_{2}(k)$. In this paper, we give some concrete examples and properties of strongly liftable schemes. As an application, we prove that the Kawamata-Viehweg vanishing theorem in positive characteristic holds on any normal projective surface which is birational to a strongly liftable surface.
\end{abstract}

\section{Introduction}

As is well known, the Kawamata-Viehweg vanishing theorem plays a crucial role in birational geometry of algebraic varieties, and it is of several forms, where the most general form is stated for log pairs which have only Kawamata log terminal singularities [KMM87, Theorem 1-2-5].

Theorem 1.1 (Kawamata-Viehweg vanishing). Let $X$ be a normal projective variety over an algebraically closed field $k$ with $\operatorname{char}(k)=0, B=\sum b_{i} B_{i}$ an effective $\mathbb{Q}$-divisor on $X$, and $D$ a $\mathbb{Q}$-Cartier Weil divisor on $X$. Assume that $(X, B)$ is Kawamata log terminal (KLT for short), and $D-\left(K_{X}+B\right)$ is ample. Then $H^{i}(X, D)=0$ holds for any $i>0$.

In what follows, we always work over an algebraically closed field $k$ of characteristic $p>0$ unless otherwise stated. The Kawamata-Viehweg vanishing theorem for smooth projective varieties in positive characteristic has first been proved by Hara [Ha98] under the lifting condition over $W_{2}(k)$ of certain log pairs.

Theorem 1.2 (Kawamata-Viehweg vanishing in char. $p>0$ ). Let $X$ be a smooth projective variety over $k$ of dimension $d, H$ an ample $\mathbb{Q}$-divisor on $X$, and $D$ a simple normal crossing divisor containing $\operatorname{Supp}(\langle H\rangle)$. Assume that $(X, D)$ admits a lifting over $W_{2}(k)$. Then

$$
H^{i}\left(X, \Omega_{X}^{j}(\log D)(-\ulcorner H\urcorner)\right)=0 \text { holds for any } i+j<\inf (d, p) .
$$

In particular, $H^{i}\left(X, K_{X}+\ulcorner H\urcorner\right)=0$ holds for any $i>d-\inf (d, p)$.

Received by the editors March 2, 2010

This paper was partially supported by the National Natural Science Foundation of China (Grant No. 10901037) and Ph.D. Programs Foundation of Ministry of Education of China (Grant No. 20090071120004). 
To obtain a practical version of the Kawamata-Viehweg vanishing theorem in positive characteristic instead of Theorem 1.2, we have to take the lifting property of log pairs into account. For this purpose, we introduce the following notion. A smooth scheme $X$ is said to be strongly liftable, if $X$ and all prime divisors on $X$ can be lifted simultaneously over $W_{2}(k)$ (see Definition 2.3 for more details).

First of all, we can give many concrete examples of strongly liftable schemes.

Theorem 1.3. The following schemes are strongly liftable:

(i) $\mathbb{A}_{k}^{n}, \mathbb{P}_{k}^{n}$ and a smooth projective curve;

(ii) a smooth projective variety of Picard number 1 which is a complete intersection in $\mathbb{P}_{k}^{n}$;

(iii) a smooth projective rational surface;

(iv) a smooth projective surface whose relatively minimal model is $\mathbb{P}_{C}\left(\mathcal{L}_{1} \oplus \mathcal{L}_{2}\right)$, where $C$ is a smooth projective curve and $\mathcal{L}_{i}$ are invertible sheaves on $C$.

A direct consequence of Theorem 1.2 is that the Kawamata-Viehweg vanishing theorem holds on strongly liftable schemes. Furthermore, we have a stronger result in dimension two.

Theorem 1.4. The Kawamata-Viehweg vanishing theorem holds on any normal projective surface which is birational to a strongly liftable surface.

Theorems 1.3 and 1.4 imply the following consequences, where Corollary 1.5 is the main theorem of [Xie09, Theorem 1.4].

Corollary 1.5. The Kawamata-Viehweg vanishing theorem holds on rational surfaces.

Corollary 1.6. Let $Y$ be a smooth projective variety of dimension $d \geq 3$ which is a complete intersection in $\mathbb{P}_{k}^{n}, f: X \rightarrow Y$ a composition of blow-ups along closed points, and $H$ an ample $\mathbb{Q}$-divisor on $X$ with $\operatorname{Supp}(\langle H\rangle)$ being simple normal crossing. Then $H^{i}\left(X, K_{X}+\ulcorner H\urcorner\right)=0$ holds for any $i>d-\inf (d, p)$.

In $\S 2$, we will give various definitions of liftings over $W_{2}(k)$ and some preliminary results. In $\S 3$, we will give some examples and properties of strongly liftable schemes. $\S 4$ is devoted to the proof of the main results. For the necessary notions and results in birational geometry, e.g. Kawamata log terminal singularity, we refer the reader to [KMM87] and [KM98].

Notation. We use $\sim$ to denote linear equivalence, $\equiv$ to denote numerical equivalence, and $[B]=\sum\left[b_{i}\right] B_{i}$ (resp. $\ulcorner B\urcorner=\sum\left\ulcorner b_{i}\right\urcorner B_{i},\langle B\rangle=\sum\left\langle b_{i}\right\rangle B_{i},\{B\}=\sum\left\{b_{i}\right\} B_{i}$ ) to denote the round-down (resp. round-up, fractional part, upper fractional part) of a $\mathbb{Q}$-divisor $B=\sum b_{i} B_{i}$, where for a real number $b,[b]:=\max \{n \in \mathbb{Z} \mid n \leq b\}$, $\ulcorner b\urcorner:=-[-b],\langle b\rangle:=b-[b]$ and $\{b\}:=\ulcorner b\urcorner-b$.

\section{Preliminaries}

Let us first recall some definitions from [EV92, Definition 8.11].

Definition 2.1. Let $W_{2}(k)$ be the ring of Witt vectors of length two of $k$. Then $W_{2}(k)$ is flat over $\mathbb{Z} / p^{2} \mathbb{Z}$, and $W_{2}(k) \otimes_{\mathbb{Z} / p^{2} \mathbb{Z}} \mathbb{F}_{p}=k$. For the explicit construction 
and further properties of $W_{2}(k)$, we refer the reader to [Se62, II.6]. The following definition generalizes the definition [DI87, 1.6] of liftings of $k$-schemes over $W_{2}(k)$.

Let $X$ be a noetherian scheme over $k$, and $D=\sum D_{i}$ a reduced Cartier divisor on $X$. A lifting of $(X, D)$ over $W_{2}(k)$ consists of a scheme $\widetilde{X}$ and closed subschemes $\widetilde{D_{i}} \subset \widetilde{X}$, all defined and flat over $W_{2}(k)$ such that $X=\widetilde{X} \times_{\operatorname{Spec} W_{2}(k)} \operatorname{Spec} k$ and $D_{i}=\widetilde{D_{i}} \times \times_{\text {Spec } W_{2}(k)}$ Spec $k$. We write $\widetilde{D}=\sum \widetilde{D_{i}}$ and say that $(\widetilde{X}, \widetilde{D})$ is a lifting of $(X, D)$ over $W_{2}(k)$, if no confusion is likely.

In the above definition, assume further that $X$ is smooth over $k$ and $D=\sum D_{i}$ is simple normal crossing. If $(\widetilde{X}, \widetilde{D})$ is a lifting of $(X, D)$ over $W_{2}(k)$, then $\widetilde{X}$ is smooth over $W_{2}(k)$ and $\widetilde{D}=\sum \widetilde{D_{i}}$ is relatively simple normal crossing over $W_{2}(k)$, i.e. $\widetilde{X}$ is covered by affine open subsets $\left\{U_{\alpha}\right\}$, such that each $U_{\alpha}$ is étale over $\mathbb{A}_{W_{2}(k)}^{n}$ via coordinates $\left\{x_{1}, \cdots, x_{n}\right\}$ and $\left.\widetilde{D}\right|_{U_{\alpha}}$ is defined by the equation $x_{1} \cdots x_{\nu}=0$ with $1 \leq \nu \leq n$ (see [EV92, Lemmas 8.13, 8.14]).

If $\widetilde{X}$ is a lifting of $X$ over $W_{2}(k)$, then there is an exact sequence of $\mathcal{O}_{\tilde{X}}$-modules:

$$
0 \rightarrow \mathcal{O}_{X} \stackrel{p}{\rightarrow} \mathcal{O}_{\widetilde{X}} \stackrel{r}{\rightarrow} \mathcal{O}_{X} \rightarrow 0,
$$

where $p(x):=p x$ and $r(\widetilde{x}):=\widetilde{x} \bmod p$ for $x \in \mathcal{O}_{X}, \widetilde{x} \in \mathcal{O}_{\widetilde{X}}$ (see [EV92, Lemma $8.13])$.

Definition 2.2. Let $X$ be a smooth scheme over $k, D=\sum D_{i}$ a reduced divisor on $X$, and $Z$ a closed subscheme of $X$ smooth over $k$ of codimension $s \geq 2$. A mixed lifting of $(X, D+Z)$ over $W_{2}(k)$ consists of a smooth scheme $\widetilde{X}$ over $W_{2}(k)$, closed subschemes $\widetilde{D_{i}} \subset \widetilde{X}$ flat over $W_{2}(k)$, and a closed subscheme $\widetilde{Z} \subset \widetilde{X}$ smooth over $W_{2}(k)$ such that $X=\widetilde{X} \times_{\operatorname{Spec} W_{2}(k)} \operatorname{Spec} k, D_{i}=\widetilde{D}_{i} \times_{\operatorname{Spec} W_{2}(k)} \operatorname{Spec} k$ and $Z=\widetilde{Z} \times_{\text {Spec } W_{2}(k)}$ Spec $k$. We write $\widetilde{D}=\sum \widetilde{D_{i}}$ and say that $(\widetilde{X}, \widetilde{D}+\widetilde{Z})$ is a mixed lifting of $(X, D+Z)$ over $W_{2}(k)$, if no confusion is likely.

In the above definition, either $D=\emptyset$ or $Z=\emptyset$ is allowed. Obviously, if $Z=\emptyset$ then a mixed lifting $(\widetilde{X}, \widetilde{D})$ of $(X, D)$ is indeed a lifting of $(X, D)$ over $W_{2}(k)$.

Definition 2.3. Let $X$ be a smooth scheme over $k$. $X$ is said to be strongly liftable over $W_{2}(k)$, if the following two conditions hold:

(i) $X$ is liftable over $W_{2}(k)$;

(ii) there is a lifting $\widetilde{X}$ of $X$, such that for any prime divisor $D$ on $X,(X, D)$ has a lifting $(\widetilde{X}, \widetilde{D})$ over $W_{2}(k)$ in the sense of Definition 2.1.

Therefore, for a strongly liftable smooth scheme $X$, both $X$ and any effective divisor on $X$ can be lifted simultaneously over $W_{2}(k)$.

Lemma 2.4. Let $X$ be a smooth scheme over $k, D$ a reduced divisor on $X$, and $Z \subset X$ a closed subscheme smooth over $k$ of codimension $s \geq 2$. Let $\pi: X^{\prime} \rightarrow X$ be the blow-up of $X$ along $Z$ with the exceptional divisor $E, D^{\prime}=\pi_{*}^{-1} D$ the strict transform of $D$. Assume that $(X, D+Z)$ admits a mixed lifting over $W_{2}(k)$. Then $\left(X^{\prime}, D^{\prime}+E\right)$ admits a mixed lifting over $W_{2}(k)$.

Proof. Let $(\widetilde{X}, \widetilde{D}+\widetilde{Z})$ be a mixed lifting of $(X, D+Z)$ over $W_{2}(k)$. Then $\widetilde{Z} \subset \widetilde{X}$ is a closed subscheme smooth over $W_{2}(k)$ of codimension $s \geq 2$. Let $\widetilde{I}$ be the ideal 
sheaf of $\widetilde{Z}$ in $\tilde{X}, \widetilde{\pi}: \widetilde{X}^{\prime} \rightarrow \widetilde{X}$ the blow-up of $\widetilde{X}$ along $\widetilde{Z}$ with the exceptional divisor $\widetilde{E}$, and $\widetilde{D}^{\prime}=\widetilde{\pi}_{*}^{-1} \widetilde{D}$. By [Ha77, Corollary II.7.15], we have the following commutative diagram:

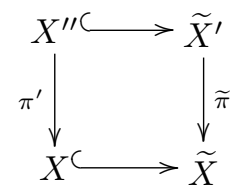

where $\pi^{\prime}: X^{\prime \prime} \rightarrow X$ is the blow-up of $X$ with respect to the ideal sheaf $\widetilde{I} \otimes_{W_{2}(k)} k=I$, the ideal sheaf of $Z$ in $X$. Hence $X^{\prime \prime}=X^{\prime}$ and $\pi^{\prime}=\pi$. Since $\widetilde{X}$ is smooth over $W_{2}(k)$, so is $\widetilde{X}^{\prime}$. Note that $\widetilde{X}^{\prime} \times_{\operatorname{Spec} W_{2}(k)} \operatorname{Spec} k=\operatorname{Proj}\left(\oplus_{i} \widetilde{I}^{i}\right) \times_{\operatorname{Spec}} W_{2}(k) \operatorname{Spec} k=$ $\operatorname{Proj}\left(\oplus_{i} \widetilde{I}^{i} \otimes_{W_{2}(k)} k\right)=\operatorname{Proj}\left(\oplus_{i} I^{i}\right)=X^{\prime}$, so $\widetilde{X}^{\prime}$ is a lifting of $X^{\prime}$ over $W_{2}(k)$. It is easy to see that $\widetilde{D}^{\prime} \times$ Spec $W_{2}(k)$ Spec $k=D^{\prime}$ and $\widetilde{E} \times$ Spec $W_{2}(k)$ Spec $k=E$, hence $\left(X^{\prime}, D^{\prime}+E\right)$ has a mixed lifting $\left(\widetilde{X}^{\prime}, \widetilde{D}^{\prime}+\widetilde{E}\right)$ over $W_{2}(k)$.

Lemma 2.5. Let $X$ be a smooth scheme over $k$, and $P \in X$ a closed point. If $X$ has a lifting over $W_{2}(k)$, then $(X, P)$ has a mixed lifting over $W_{2}(k)$.

Proof. Let $\tilde{X}$ be a lifting of $X$ over $W_{2}(k), X \hookrightarrow \widetilde{X}$ the induced closed immersion, and $\eta: \widetilde{X} \rightarrow$ Spec $W_{2}(k)$ the structure morphism. Let Spec $k \hookrightarrow X$ be the closed immersion associated to the closed point $P \in X$, and $\operatorname{Spec} k \hookrightarrow \operatorname{Spec} W_{2}(k)$ the natural closed immersion. We have the following commutative square:

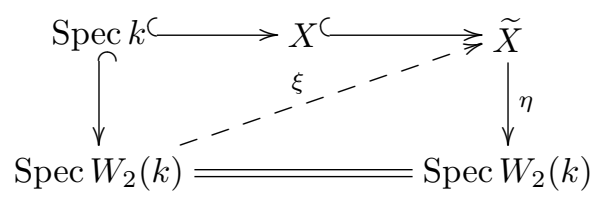

Since Spec $k \hookrightarrow$ Spec $W_{2}(k)$ is a closed immersion with ideal sheaf square zero and $\eta: \widetilde{X} \rightarrow \operatorname{Spec} W_{2}(k)$ is smooth, there is a morphism $\xi: \operatorname{Spec} W_{2}(k) \rightarrow \widetilde{X}$ such that the induced diagrams are commutative. Since $\xi$ is a section of $\eta$, it defines a closed subscheme $\widetilde{P} \subset \widetilde{X}$ smooth over $W_{2}(k)$. It follows from the upper commutativity that $P=\widetilde{P} \times{ }_{\text {Spec } W_{2}(k)}$ Spec $k$ holds. Therefore, $(\widetilde{X}, \widetilde{P})$ is a mixed lifting of $(X, P)$ over $W_{2}(k)$.

Proposition 2.6. Let $X$ be a smooth scheme over $k$ with $\operatorname{dim} X \geq 2, P \in X$ a closed point, and $\pi: X^{\prime} \rightarrow X$ the blow-up of $X$ along $P$. If $X$ is strongly liftable (resp. liftable) over $W_{2}(k)$, then $X^{\prime}$ is strongly liftable (resp. liftable) over $W_{2}(k)$.

Proof. For liftability, it follows from Lemmas 2.5 and 2.4. For strong liftability, let $\widetilde{X}$ be a lifting of $X$ satisfying the condition (ii) in Definition 2.3. Let $\widetilde{P} \in \widetilde{X}$ be a mixed lifting of $P \in X$ as in Lemma 2.5, and $\pi: \widetilde{X}^{\prime} \rightarrow \widetilde{X}$ the blow-up of $\widetilde{X}$ along $\widetilde{P}$. A similar argument to the proof of Lemma 2.4 shows that $\widetilde{X}^{\prime}$ is a lifting of $X^{\prime}$. Therefore, it suffices to show that $\widetilde{X}^{\prime}$ satisfies the condition (ii) in Definition 2.3. For any prime divisor $D^{\prime}$ on $X^{\prime}$, either $\pi_{*}\left(D^{\prime}\right)$ is a prime divisor or $\pi_{*}\left(D^{\prime}\right)=P$. If $\pi_{*}\left(D^{\prime}\right)=D$ is a prime divisor, then $(X, D+P)$ has a mixed lifting $(\widetilde{X}, \widetilde{D}+\widetilde{P})$ by assumption, hence $\left(X^{\prime}, D^{\prime}\right)$ has a lifting $\left(\widetilde{X}^{\prime}, \widetilde{D}^{\prime}\right)$ by Lemma 2.4. If $\pi_{*}\left(D^{\prime}\right)=P$, 
i.e. $D^{\prime}$ is the exceptional divisor $E$ of $\pi$, then via the mixed lifting $(\widetilde{X}, \widetilde{P})$ of $(X, P)$, $\left(X^{\prime}, E\right)$ has a lifting $\left(\widetilde{X}^{\prime}, \widetilde{E}\right)$ by Lemma 2.4 .

\section{Examples of strongly liftable schemes}

Lemma 3.1. $\mathbb{A}_{k}^{n}$ and $\mathbb{P}_{k}^{n}$ are strongly liftable over $W_{2}(k)$.

Proof. Any prime divisor $D$ on $\mathbb{A}_{k}^{n}$ (resp. $\mathbb{P}_{k}^{n}$ ) is defined by the equation $f=0$, where $f$ is a polynomial in $k\left[x_{1}, \cdots, x_{n}\right]$ (resp. a homogeneous polynomial in $k\left[x_{0}, \cdots, x_{n}\right]$ ). Therefore, we can lift each coefficient of $f$ over $W_{2}(k)$ to obtain a polynomial $\tilde{f}$ in $W_{2}(k)\left[x_{1}, \cdots, x_{n}\right]$ (resp. a homogeneous polynomial $\tilde{f}$ in $W_{2}(k)\left[x_{0}, \cdots, x_{n}\right]$ ). Then the divisor $\widetilde{D}$ defined by $\widetilde{f}=0$ is a lifting of $D$ over $W_{2}(k)$.

Lemma 3.2. Any smooth projective curve is strongly liftable over $W_{2}(k)$.

Proof. It follows from [I196, Proposition 2.12] that for any smooth scheme $X$ over $k$, there is an obstruction $o(X) \in \operatorname{Ext}_{\mathcal{O}_{X}}^{2}\left(\Omega_{X}^{1}, \mathcal{O}_{X}\right)=H^{2}\left(X, \mathcal{T}_{X}\right)$ to the liftability of $X$ over $W_{2}(k)$, i.e. $o(X)=0$ if and only if $X$ is liftable over $W_{2}(k)$. Any smooth projective curve $C$ has a lifting $\widetilde{C}$ over $W_{2}(k)$ since $H^{2}\left(C, \mathcal{T}_{C}\right)=0$. Fix such a lifting $\widetilde{C}$, then for any closed point $P \in C,(C, P)$ has a lifting $(\widetilde{C}, \widetilde{P})$ by Lemma 2.5 .

Lemma 3.3. Let $X$ be a smooth projective variety of Picard number 1 which is a complete intersection in $\mathbb{P}_{k}^{n}$. Then $X$ is strongly liftable over $W_{2}(k)$.

Proof. In fact, if $X \subset \mathbb{P}_{k}^{n}$ is a complete intersection with $\operatorname{dim} X \geq 3$, then the Picard group $\operatorname{Pic}(X)$ is the free abelian group generated by the isomorphism class of the invertible sheaf $\mathcal{O}_{X}(1)$, which implies automatically the Picard number $\rho(X)=1$ (see [SGA 2, XII, Corollaire 3.7]). If $\operatorname{dim} X=2$, then [SGA 7, XI, Théorème 1.8] says that $\operatorname{Pic}(X)$ is torsion-free and $\mathcal{O}_{X}(1)$ is not divisible in $\operatorname{Pic}(X)$, hence $\operatorname{Pic}(X)$ is the free abelian group generated by the isomorphism class of $\mathcal{O}_{X}(1)$ since $\rho(X)=1$.

Let $\operatorname{dim} X=d$, and $f_{1}, \cdots, f_{n-d}$ homogeneous polynomials in $k\left[x_{0}, \cdots, x_{n}\right]$ such that $X=\operatorname{Proj} k\left[x_{0}, \cdots, x_{n}\right] /\left(f_{1}, \cdots, f_{n-d}\right)$. Let $D$ be an irreducible divisor on $X$. Then $\mathcal{O}_{X}(D) \cong \mathcal{O}_{X}(r)$ with $r \geq 1$. First of all, by lifting each coefficient of $f_{i}$, we can take $\widetilde{f}_{1}, \cdots, \widetilde{f}_{n-d} \in W_{2}(k)\left[x_{0}, \cdots, x_{n}\right]$ lifting $f_{1}, \cdots, f_{n-d}$ respectively. The scheme $\widetilde{X}$ defined by $\operatorname{Proj} W_{2}(k)\left[x_{0}, \cdots, x_{n}\right] /\left(\widetilde{f}_{1}, \cdots, \widetilde{f}_{n-d}\right)$ is a lifting of $X$.

Since $X$ is a smooth subscheme of $\mathbb{P}^{n}$, the sequence $\left\{f_{1}, \cdots, f_{n-d}\right\}$ is regular, which gives rise to the following Koszul resolution of the sheaf $\mathcal{O}_{X}$ :

$$
0 \rightarrow \wedge^{n-d} \mathcal{M} \rightarrow \wedge^{n-d-1} \mathcal{M} \rightarrow \cdots \rightarrow \wedge^{1} \mathcal{M} \rightarrow \mathcal{O}_{\mathbb{P}^{n}} \rightarrow \mathcal{O}_{X} \rightarrow 0,
$$

where $\mathcal{M}=\mathcal{O}_{\mathbb{P}^{n}}^{\oplus n-d}$. The exact sequence (2) factorizes into short exact sequences:

$$
0 \rightarrow \mathcal{I}_{i} \rightarrow \wedge^{i-1} \mathcal{M} \rightarrow \mathcal{I}_{i-1} \rightarrow 0,
$$

where $\mathcal{I}_{0}=\mathcal{O}_{X}, \mathcal{I}_{n-d}=\wedge^{n-d} \mathcal{M}$ and $\mathcal{I}_{1}, \cdots, \mathcal{I}_{n-d-1}$ are defined by (2). We will use frequently the vanishing $H^{j}\left(\mathbb{P}^{n}, \wedge^{i} \mathcal{M}(r)\right)=0$ for any $i \geq 0, j>0$ and $r \geq 1$, which follows from the vanishing $H^{j}\left(\mathbb{P}^{n}, \mathcal{O}_{\mathbb{P}}(r)\right)=0$ for any $j>0$ and $r \geq 1$. To prove that $H^{0}\left(\mathbb{P}^{n}, \mathcal{O}_{\mathbb{P}^{n}}(r)\right) \rightarrow H^{0}\left(X, \mathcal{O}_{X}(D)\right)$ is surjective, it suffices to show $H^{1}\left(\mathbb{P}^{n}, \mathcal{I}_{1}(r)\right)=0$. By using the exact sequence (3) and the above vanishing, the induction reduces to show $H^{n-d}\left(\mathbb{P}^{n}, \mathcal{I}_{n-d}(r)\right)=0$, which follows from the above vanishing again. 
The surjectivity of $H^{0}\left(\mathbb{P}^{n}, \mathcal{O}_{\mathbb{P}^{n}}(r)\right) \rightarrow H^{0}\left(X, \mathcal{O}_{X}(D)\right)$ implies that we can take a hypersurface $H \subset \mathbb{P}^{n}$ of degree $r$ such that $D=X \cap H$. Take a lifting $\widetilde{H}$ of $H$ as in Lemma 3.1, and define $\widetilde{D}=\widetilde{X} \cap \widetilde{H}$. Then it is easy to see that $\widetilde{D} \subset \widetilde{X}$ is a lifting of $D \subset X$.

Let $X$ be a smooth projective scheme over $k, \widetilde{X}$ a lifting of $X$ over $W_{2}(k)$, and $\iota: X \hookrightarrow \widetilde{X}$ the closed immersion. We have a natural group homomorphism $\iota^{*}$ : $\operatorname{Pic}(\widetilde{X}) \rightarrow \operatorname{Pic}(X)$ defined by $\widetilde{\mathcal{L}} \mapsto \iota^{*} \widetilde{\mathcal{L}}$. There is an exact sequence of abelian sheaves:

$$
0 \rightarrow \mathcal{O}_{X} \stackrel{q}{\rightarrow} \mathcal{O}_{\widetilde{X}}^{*} \stackrel{r}{\rightarrow} \mathcal{O}_{X}^{*} \rightarrow 1,
$$

where $q(x):=1+p x, r(\widetilde{x}):=\widetilde{x} \bmod p$ for $x \in \mathcal{O}_{X}, \widetilde{x} \in \mathcal{O}_{\tilde{X}}$, which gives rise to an exact sequence:

$$
H^{1}\left(\widetilde{X}, \mathcal{O}_{\widetilde{X}}^{*}\right) \stackrel{\pi}{\rightarrow} H^{1}\left(X, \mathcal{O}_{X}^{*}\right) \rightarrow H^{2}\left(X, \mathcal{O}_{X}\right)
$$

We can identify $\iota^{*}: \operatorname{Pic}(\widetilde{X}) \rightarrow \operatorname{Pic}(X)$ with $\pi: H^{1}\left(\widetilde{X}, \mathcal{O}_{\widetilde{X}}^{*}\right) \rightarrow H^{1}\left(X, \mathcal{O}_{X}^{*}\right)$, by using the canonical isomorphisms $H^{1}\left(X, \mathcal{O}_{X}^{*}\right) \stackrel{\sim}{\rightarrow} \operatorname{Pic}(X)$ and $H^{1}\left(\widetilde{X}, \mathcal{O}_{\widetilde{X}}^{*}\right) \stackrel{\sim}{\rightarrow} \operatorname{Pic}(\widetilde{X})$. Let $D$ be a prime divisor on $X$, and $\mathcal{L}_{D}=\mathcal{O}_{X}(D)$ the associated invertible sheaf on $X$. Assume that $\iota^{*}: \operatorname{Pic}(\widetilde{X}) \rightarrow \operatorname{Pic}(X)$ is surjective. Then $\mathcal{L}_{D}$ has a lifting $\widetilde{\mathcal{L}}_{D}$ on $\widetilde{X}$. Furthermore, by tensoring the exact sequence (1) with $\widetilde{\mathcal{L}}_{D}$, we have a natural exact sequence of sheaves of $\mathcal{O}_{\tilde{X}}$-modules:

$$
0 \rightarrow \mathcal{L}_{D} \stackrel{p}{\rightarrow} \widetilde{\mathcal{L}}_{D} \stackrel{r}{\rightarrow} \mathcal{L}_{D} \rightarrow 0
$$

which gives rise to an exact sequence:

$$
H^{0}\left(\widetilde{X}, \widetilde{\mathcal{L}}_{D}\right) \stackrel{\pi_{D}}{\longrightarrow} H^{0}\left(X, \mathcal{L}_{D}\right) \rightarrow H^{1}\left(X, \mathcal{L}_{D}\right) .
$$

Proposition 3.4. Let $X$ be a smooth projective scheme over $k$, and $\tilde{X}$ a lifting of $X$ over $W_{2}(k)$. We use the same notations as above. Then $X$ is strongly liftable if and only if the following two conditions hold:

(i) $\pi: H^{1}\left(\widetilde{X}, \mathcal{O}_{\widetilde{X}}^{*}\right) \rightarrow H^{1}\left(X, \mathcal{O}_{X}^{*}\right)$ is surjective;

(ii) $\pi_{D}: H^{0}\left(\widetilde{X}, \widetilde{\mathcal{L}}_{D}\right) \rightarrow H^{0}\left(X, \mathcal{L}_{D}\right)$ is surjective for any prime divisor $D$ on $X$.

Proof. Sufficiency: For any prime divisor $D$ on $X, D$ corresponds to a nonzero section $s \in H^{0}\left(X, \mathcal{L}_{D}\right)$ with $D=\operatorname{div}_{0}(s)$. Since $\pi$ is surjective, we can take a lifting $\widetilde{\mathcal{L}}_{D}$ of $\mathcal{L}_{D}$ and consider $\pi_{D}: H^{0}\left(\widetilde{X}, \widetilde{\mathcal{L}}_{D}\right) \rightarrow H^{0}\left(X, \mathcal{L}_{D}\right)$. Since $\pi_{D}$ is surjective, there is a nonzero section $\widetilde{s} \in H^{0}\left(\widetilde{X}, \widetilde{\mathcal{L}}_{D}\right)$. Let $\widetilde{D}=\operatorname{div}_{0}(\widetilde{s})$. Then it is easy to see that $(\widetilde{X}, \widetilde{D})$ is a lifting of $(X, D)$ over $W_{2}(k)$.

Necessity: Since $X$ is strongly liftable, any effective divisor $D \subset X$ has a lifting $\widetilde{D} \subset \widetilde{X}$. For any invertible sheaf $\mathcal{L}$ on $X$, write $\mathcal{L}=\mathcal{O}_{X}\left(D_{1}-D_{2}\right)$ with $D_{i}$ effective. Lift $D_{i}$ to $\widetilde{D}_{i}$ for $i=1,2$. Then the invertible sheaf $\widetilde{\mathcal{L}}:=\mathcal{O}_{\widetilde{X}}\left(\widetilde{D}_{1}-\widetilde{D}_{2}\right)$ lifts $\mathcal{L}$, which proves the condition (i). The condition (ii) is obvious.

Corollary 3.5. Let $X$ be a smooth projective scheme over $k$, and $\widetilde{X}$ a lifting of $X$ over $W_{2}(k)$. We use the same notations as above. Then $X$ is strongly liftable if the following two conditions hold:

(i) $H^{2}\left(X, \mathcal{O}_{X}\right)=0$; 
(ii) for any prime divisor $D$ on $X$, either $\pi_{D}: H^{0}\left(\widetilde{X}, \widetilde{\mathcal{L}}_{D}\right) \rightarrow H^{0}\left(X, \mathcal{L}_{D}\right)$ is surjective or $H^{1}\left(X, \mathcal{O}_{X}(D)\right)=0$.

Proof. From the exact sequence (5), it follows that if $H^{2}\left(X, \mathcal{O}_{X}\right)=0$ then $\pi$ : $H^{1}\left(\widetilde{X}, \mathcal{O}_{\widetilde{X}}^{*}\right) \rightarrow H^{1}\left(X, \mathcal{O}_{X}^{*}\right)$ is surjective. From the exact sequence $(7)$, it follows that if $H^{1}\left(X, \mathcal{O}_{X}(D)\right)=0$ then $\pi_{D}: H^{0}\left(\widetilde{X}, \widetilde{\mathcal{L}}_{D}\right) \rightarrow H^{0}\left(X, \mathcal{L}_{D}\right)$ is surjective.

Lemma 3.6. Let $S$ be a scheme over $k$, and $\widetilde{S}$ a lifting of $S$ over $W_{2}(k)$. Let $X$ be a smooth $S$-scheme, and $D \subset X$ a divisor which is relatively simple normal crossing over $S$. Then there is an obstruction $o(X / S, D) \in \operatorname{Ext}_{\mathcal{O}_{X}}^{2}\left(\Omega_{X / S}^{1}(\log D), \mathcal{O}_{X}\right)=$ $H^{2}\left(X, \mathcal{T}_{X / S}(-\log D)\right)$ to the liftability of $(X, D)$ over $\widetilde{S}$, i.e. $o(X / S, D)=0$ if and only if $(X, D)$ is liftable over $\widetilde{S}$.

Proof. The case when $D=\emptyset$ was verified directly in [I196, Proposition 2.12]. For the general case, [EV92, Proposition 8.22] just showed that the isomorphisms of liftings of $(X, D)$ over $\widetilde{S}$ form a torsor under the group $\operatorname{Hom}_{\mathcal{O}_{X}}\left(\Omega_{X / S}^{1}(\log D), \mathcal{O}_{X}\right)$, hence by a similar argument to that of [I196, Proposition 2.12], we get the required obstruction $o(X / S, D) \in \operatorname{Ext}_{\mathcal{O}_{X}}^{2}\left(\Omega_{X / S}^{1}(\log D), \mathcal{O}_{X}\right)=H^{2}\left(X, \mathcal{T}_{X / S}(-\log D)\right)$.

Lemma 3.7. Let $C$ be a smooth projective curve, and $\mathcal{E}$ a locally free sheaf on $C$ of rank 2. Let $X=\mathbb{P}(\mathcal{E}), f: X \rightarrow C$ the natural projection, and $E$ a section of $f$. Then $f:(X, E) \rightarrow C$ has a lifting $\widetilde{f}:(\widetilde{X}, \widetilde{E}) \rightarrow \widetilde{C}$ over $W_{2}(k)$.

Proof. Let $\widetilde{C}$ be a lifting of $C$. By Lemma 3.6, the obstruction to the liftability of $(X, E)$ over $\widetilde{C}$ is $o(X / C, E) \in \operatorname{Ext}_{\mathcal{O}_{X}}^{2}\left(\Omega_{X / C}^{1}(\log E), \mathcal{O}_{X}\right)=H^{2}\left(X, \mathcal{T}_{X / C}(-\log E)\right)$. By Serre duality, $H^{2}\left(X, \mathcal{T}_{X / C}(-\log E)\right)^{\vee}=H^{0}\left(X, \Omega_{X / C}^{1}(\log E) \otimes \omega_{X}\right)=H^{0}\left(X, \omega_{X / C} \otimes\right.$ $\left.\omega_{X} \otimes \mathcal{O}_{X}(E)\right)$, which vanishes by an easy calculation.

Proposition 3.8. Let $C$ be a smooth projective curve, $\mathcal{G}$ an invertible sheaf on $C$, and $\mathcal{E}=\mathcal{O}_{C} \oplus \mathcal{G}$. Let $X=\mathbb{P}(\mathcal{E})$, and $f: X \rightarrow C$ the natural projection. Then $X$ is strongly liftable over $W_{2}(k)$.

Proof. Let $E$ be a section of $f$ with $\mathcal{O}_{X}(E) \cong \mathcal{O}_{X}(1)$. By Lemma 3.7, we can take and fix such a lifting $\widetilde{f}:(\widetilde{X}, \widetilde{E}) \rightarrow \widetilde{C}$ of $f:(X, E) \rightarrow C$. Since $H^{2}\left(X, \mathcal{O}_{X}\right)=0$, by Corollary 3.5, to prove the strong liftability of $X$, it suffices to show that for any irreducible curve $D$ on $X, \pi_{D}: H^{0}\left(\widetilde{X}, \widetilde{\mathcal{L}}_{D}\right) \rightarrow H^{0}\left(X, \mathcal{L}_{D}\right)$ is surjective, where $\mathcal{L}_{D}=\mathcal{O}_{X}(D)$.

Write $D \sim a E+f^{*} H$, where $a \geq 0$ and $H$ is a divisor on $C$. Let $\widetilde{H}$ be a divisor on $\widetilde{C}$ lifting $H, \mathcal{H}=\mathcal{O}_{X}(H)$ and $\widetilde{\mathcal{H}}=\mathcal{O}_{\widetilde{X}}(\widetilde{H})$. Then $\mathcal{L}_{D}=\mathcal{O}_{X}(D)$ has a lifting $\widetilde{\mathcal{L}}_{D}=\mathcal{O}_{\widetilde{X}}(a \widetilde{E}+\widetilde{f} * \widetilde{H})$. Note that

$$
\begin{aligned}
& H^{0}\left(X, \mathcal{L}_{D}\right)=H^{0}\left(C, f_{*} \mathcal{O}_{X}\left(a E+f^{*} H\right)\right)=H^{0}\left(C, S^{a}(\mathcal{E}) \otimes \mathcal{H}\right)=\oplus_{i=0}^{a} H^{0}\left(C, \mathcal{G}^{i} \otimes \mathcal{H}\right), \\
& H^{0}\left(\widetilde{X}, \widetilde{\mathcal{L}}_{D}\right)=H^{0}\left(\widetilde{C}, \widetilde{f}_{*} \mathcal{O}_{\widetilde{X}}\left(a \widetilde{E}+\widetilde{f}^{*} \widetilde{H}\right)\right)=H^{0}\left(\widetilde{C}, S^{a}(\widetilde{\mathcal{E}}) \otimes \widetilde{\mathcal{H}}\right)=\oplus_{i=0}^{a} H^{0}\left(\widetilde{C}, \widetilde{\mathcal{G}}^{i} \otimes \widetilde{\mathcal{H}}\right) .
\end{aligned}
$$

Therefore, $\pi_{D}: H^{0}\left(\widetilde{X}, \widetilde{\mathcal{L}}_{D}\right) \rightarrow H^{0}\left(X, \mathcal{L}_{D}\right)$ factorizes into $\pi_{D}^{i}: H^{0}\left(\widetilde{C}, \widetilde{\mathcal{G}}^{i} \otimes \widetilde{\mathcal{H}}\right) \rightarrow$ $H^{0}\left(C, \mathcal{G}^{i} \otimes \mathcal{H}\right)$. Since $C$ is strongly liftable by Lemma $3.2, \pi_{D}^{i}$ are surjective for all $i$, hence $\pi_{D}$ is surjective. 
Remark 3.9. We can generalize Proposition 3.8 as follows by an analogous proof. Let $Y$ be a strongly liftable smooth projective variety, $\mathcal{E}$ a decomposable locally free sheaf on $Y$, and $X=\mathbb{P}(\mathcal{E})$. Then $X$ is strongly liftable over $W_{2}(k)$.

Remark 3.10. Let $C$ be a smooth projective curve, $\mathcal{E}$ a locally free sheaf on $C$ of rank 2 , and $X=\mathbb{P}(\mathcal{E})$. If $\mathcal{E}$ is indecomposable, then $X$ is not necessarily strongly liftable. Such an example has been given in [Xie07, Theorem 3.1]. More precisely, if $C$ is a Tango curve, then there is an indecomposable locally free sheaf $\mathcal{E}$ on $C$ of rank 2 , such that the pull-back $F^{*} \mathcal{E}$ by the Frobenius $F: C \rightarrow C$ is decomposable, which gives rise to an irreducible curve $C^{\prime}$ on $X=\mathbb{P}(\mathcal{E})$, such that $\left(X, C^{\prime}\right)$ cannot be lifted over $W_{2}(k)$. It is also an example of liftable but not strongly liftable scheme such that the condition (ii) in Proposition 3.4 is not satisfied.

Remark 3.11. An abelian variety is another example of liftable but not strongly liftable scheme such that the condition (i) in Proposition 3.4 is not satisfied. We proceed the following argument provided by Oort. Let $(X, \mathcal{L})$ be a polarized abelian variety of dimension $g$. Then the universal deformation space of $X$, say for $\operatorname{Spec}(R)$, is smooth over $W(k)$ of relative dimension $g^{2}$, where $W(k)$ is the ring of Witt vectors of $k$, and the universal deformation space of $(X, \mathcal{L})$, say for $\operatorname{Spec}(R / I)$, is smooth over $W(k)$ of relative dimension $<g^{2}$ (see [Oo71, Oo79] for more details). Therefore, we can take a ring homomorphism $\varphi: R \rightarrow W_{2}(k)$ which induces the identity map on residue closed fields and satisfies $\varphi(I) \neq 0$. The existence of such $\varphi$ means that $X$ can be lifted over $W_{2}(k)$, while $(X, \mathcal{L})$ cannot be lifted over $W_{2}(k)$ at the same time.

\section{Proof of the main results}

Proof of Theorem 1.3. (i) follows from Lemmas 3.1 and 3.2. (ii) follows from Lemma 3.3. Both (iii) and (iv) follow from Propositions 3.8 and 2.6 since any locally free sheaf on $\mathbb{P}^{1}$ is decomposable.

The following vanishing result [KK, Corollary 2.2.5] is useful, which holds in arbitrary characteristic.

Lemma 4.1. Let $h: Y \rightarrow X$ be a proper birational morphism between normal surfaces with $Y$ smooth and with exceptional locus $E=\cup_{i=1}^{s} E_{i}$. Let $L$ be an integral divisor on $Y, 0 \leq b_{1}, \cdots, b_{s}<1$ rational numbers, and $N$ an $h$-nef $\mathbb{Q}$-divisor on $Y$. Assume $L \equiv K_{Y}+\sum_{i=1}^{s} b_{i} E_{i}+N$. Then $R^{1} h_{*} \mathcal{O}_{Y}(L)=0$ holds.

We shall prove Theorem 1.4 in the following explicit form.

Theorem 4.2. Let $X$ be a normal projective surface, $D$ a $\mathbb{Q}$-Cartier Weil divisor on $X$, and $B$ an effective $\mathbb{Q}$-divisor such that $(X, B)$ is $K L T$ and that $D-\left(K_{X}+B\right)$ is ample. If $X$ is birational to a strongly liftable smooth projective surface $Z$, then $H^{1}(X, D)=0$ holds.

Proof. Take a $\log$ resolution $h: Y \rightarrow X$ such that the following three conditions hold:

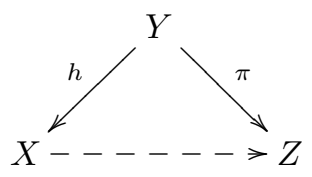


(i) $Y$ is a smooth projective surface over $k$, and we can write $K_{Y}+h_{*}^{-1} B \equiv$ $h^{*}\left(K_{X}+B\right)+\sum_{i} a_{i} E_{i}$, where $E_{i}$ are the exceptional curves of $h$ and $a_{i}>-1$ for all $i$

(ii) $G=\operatorname{Supp}\left(h_{*}^{-1} B\right) \cup \operatorname{Exc}(h)$ is simple normal crossing;

(iii) $\pi: Y \rightarrow Z$ is a birational morphism.

Let $D_{Y}=\left\ulcorner h^{*} D+\sum_{i} a_{i} E_{i}\right\urcorner$. Since $\left\ulcorner\sum_{i} a_{i} E_{i}\right\urcorner \geq 0$ is supported by $\operatorname{Exc}(h)$, we have $h_{*} \mathcal{O}_{Y}\left(D_{Y}\right)=\mathcal{O}_{X}(D)$ by the projection formula. Since $\left\{h^{*} D+\sum_{i} a_{i} E_{i}\right\}$ is supported by $\operatorname{Exc}(h)$, we can take $0<\delta_{i} \ll 1$ such that

(i) $\left[h_{*}^{-1} B+\left\{h^{*} D+\sum_{i} a_{i} E_{i}\right\}+\sum_{i} \delta_{i} E_{i}\right]=0$.

(ii) $D_{Y}-\left(K_{Y}+h_{*}^{-1} B+\left\{h^{*} D+\sum_{i} a_{i} E_{i}\right\}+\sum_{i} \delta_{i} E_{i}\right) \equiv h^{*}\left(D-\left(K_{X}+B\right)\right)-\sum_{i} \delta_{i} E_{i}$ is ample.

Let $B_{Y}=h_{*}^{-1} B+\left\{h^{*} D+\sum_{i} a_{i} E_{i}\right\}+\sum_{i} \delta_{i} E_{i}$. Then $H_{Y}=D_{Y}-\left(K_{Y}+B_{Y}\right)$ is ample, $\operatorname{Supp}\left(\left\langle H_{Y}\right\rangle\right)=\operatorname{Supp}\left(B_{Y}\right)$ is simple normal crossing, and $K_{Y}+\left\ulcorner H_{Y}\right\urcorner=D_{Y}$. Note that

$$
D_{Y} \equiv K_{Y}+\left\{h^{*} D+\sum_{i} a_{i} E_{i}\right\}+h^{*}\left(D-\left(K_{X}+B\right)\right)+h_{*}^{-1} B .
$$

By Lemma 4.1, we have $R^{1} h_{*} \mathcal{O}_{Y}\left(D_{Y}\right)=0$, hence $H^{1}\left(Y, D_{Y}\right)=H^{1}\left(X, h_{*} \mathcal{O}_{Y}\left(D_{Y}\right)\right)=$ $H^{1}(X, D)$.

Since $\pi: Y \rightarrow Z$ is a birational morphism between smooth projective surfaces, it is a composition of blow-ups [Ha77, Corollary V.5.4]. Since $Z$ is strongly liftable over $W_{2}(k)$, so is $Y$ by Proposition 2.6 , hence $(Y, G)$ admits a lifting over $W_{2}(k)$. Since $G$ contains $\operatorname{Supp}\left(\left\langle H_{Y}\right\rangle\right)$, we have $H^{1}(X, D)=H^{1}\left(Y, D_{Y}\right)=H^{1}\left(Y, K_{Y}+\left\ulcorner H_{Y}\right\urcorner\right)=0$ by Theorem 1.2.

Remark 4.3. It seems impossible to generalize Theorem 4.2 (i.e. Theorem 1.4) to the higher dimensional cases because of the following reasons: First of all, it is unknown whether the strong liftability is stable under the blow-ups along higher dimensional centers, while the center in Proposition 2.6 is of dimension zero. Secondly, the analogue of Lemma 4.1 fails definitely on higher dimensional varieties. Finally, the higher dimensional minimal model program is more involved, and extremal contractions and flips are more general and complicated than blow-ups or blow-downs.

Proof of Corollary 1.6. It follows from Lemma 3.3, Proposition 2.6 and Theorem 1.2.

\section{Acknowledgements}

I would like to express my gratitude to Professors Luc Illusie and Frans Oort for useful comments.

\section{References}

[DI87] P. Deligne, L. Illusie, Relèvements modulo $p^{2}$ et décomposition du complexe de de Rham, Invent. Math., 89 (1987), 247-270.

[EV92] H. Esnault, E. Viehweg, Lectures on vanishing theorems, DMV Seminar, vol. 20, Birkhäuser, 1992.

[SGA 2] A. Grothendieck, et al., Cohomologie locale des faisceaux cohérents et théorèmes de Lefschetz locale et globaux, North-Holland, 1968. 
[SGA 7] A. Grothendieck, et al., Groupes de monodromie en géométrie algébrique, Part I, Lecture Notes in Math., vol. 225, Springer-Verlag, 1972; Part II, Lecture Notes in Math., vol. 340, Springer-Verlag, 1973.

[Ha98] N. Hara, A characterization of rational singularities in terms of injectivity of Frobenius maps, Amer. J. Math., 120 (1998), 981-996.

[Ha77] R. Hartshorne, Algebraic geometry, Springer-Verlag, 1977.

[I196] L. Illusie, Frobenius et dégénérescence de Hodge, in J. Bertin, J.-P. Demailly, L. Illusie and C. Peters, Introduction à la Théorie de Hodge, Panoramas et Synthèses, vol. 3, Socité de Mathmatiques de France, Marseilles, 113-168, 1996.

[KMM87] Y. Kawamata, K. Matsuda, K. Matsuki, Introduction to the minimal model problem, Alg. Geom. Sendai 1985, Adv. Stud. Pure Math., 10 (1987), 283-360.

[KK] J. Kollár, S. Kovács, Birational geometry of log surfaces, preprint, which can be found at http://www.math.washington.edu/ kovacs/pdf/BiratLogSurf.pdf.

[KM98] J. Kollár, S. Mori, Birational geometry of algebraic varieties, Cambridge Tracts in Math., vol. 134, 1998.

[Oo71] F. Oort, Finite group schemes, local moduli for abelian varieties, and lifting problems, Compositio Math., 23 (1971), 265-296.

[Oo79] F. Oort, Abelian varieties: moduli and lifting properties, Algebraic geometry (Proc. Summer Meeting, Univ. Copenhagen, Copenhagen, 1978), 477-495, Lecture Notes in Math., vol. 732, Springer, 1979 .

[Se62] J.-P. Serre, Corps locaux, Hermann, 1962.

[Xie07] Q. Xie, Counterexamples to the Kawamata-Viehweg vanishing on ruled surfaces in positive characteristic, preprint, math.AG/0702554.

[Xie09] Q. Xie, Kawamata-Viehweg vanishing on rational surfaces in positive characteristic, to appear in Math. Zeit., arXiv:0710.2706.

School of Mathematical Sciences, Fudan University, Shanghai 200433, China

E-mail address: qhxie@fudan.edu.cn

$U R L:$ http://homepage.fudan.edu.cn/ ${ }^{\sim}$ qhxie/ 